\title{
CISTOADENOMA SEROSO OVÁRICO GIGANTE EN LA POSMENOPAUSIA. REPORTE DE UN CASO
}

\author{
Félix Dasio Ayala Peralta ${ }^{1, a}$, Luis Quiñones Vasquez $z^{2,3, b}$, Luis Sandoval Valiente ${ }^{2,3, c}$, \\ Doris Garrido Rivadeneira ${ }^{3, d}$
}

\begin{abstract}
RESUMEN
El cistoadenoma seroso de ovario es un tipo de tumor derivado del epitelio celómico superficial. Se denominan quistes ováricos gigantes aquellas que miden más de $10 \mathrm{~cm}$ de diámetro o que se extienden por encima del ombligo. En la literatura, algunos casos de quistes ováricos gigantes se han mencionado esporádicamente, especialmente en pacientes posmenopáusicas. Los tumores serosos son comúnmente benignos en $70-80 \%, 5-10 \%$ tiene límite potencial maligno, y 20 $25 \%$ son malignos. Solo el $10-20 \%$ de los casos son bilaterales. Ocurren en adultas de todas las edades, con edades medias reportadas de 40 a 60 años. Se reporta el caso de una mujer posmenopáusica de 79 años con un quiste ovárico izquierdo gigante que mide $14 \times 11 \times 7.5 \mathrm{~cm}$ y $794 \mathrm{~g}$ de peso conteniendo líquido seroso. Acude a consulta por presentar dolor pélvico tipo cólico irradiada a región lumbar desde hace 4 meses, con antecedente de hipertensión arterial crónica. Teniendo en cuenta la edad y el estado de la menopausia, se le realiza laparotomía para quistectomía izquierda sin ninguna complicación significativa. En el examen histopatológico, el quiste se confirmó como cistoadenoma seroso benigno del ovario. Tuvo evolución posoperatoria favorable.
\end{abstract}

Palabras clave: Cistoadenoma seroso; posmenopausia; quiste ovárico (Fuente: DeCS BIREME)

\section{POSTMENOPAUSAL SEROUS GIANT OVARIAN CYSTADENOMA.}

\section{A CASE REPORT}

\begin{abstract}
Serous ovarian cystadenoma is a type of tumor derived from the superficial celomic epithelium. Giant ovarian cysts are those that are more than $10 \mathrm{~cm}$ in diameter or that extend above the navel. In the literature, some cases of giant ovarian cysts have been mentioned sporadically, especially in postmenopausal patients. Serous tumors are commonly benign in $70-80 \%, 5-10 \%$ have a malignant potential limit, and $20-25 \%$ are malignant. Only $10-20 \%$ of cases are bilateral. They occur in adults of all ages, with reported mean ages of 40 to 60 years. The case of a 79 -year-old postmenopausal woman with a giant left ovarian cyst measuring $14 \times 11 \times 7.5 \mathrm{~cm}$ and $794 \mathrm{~g}$ of weight containing serous fluid is reported. He came to the clinic for presenting colic-type pelvic pain irradiated to the lumbar region for 4 months with a history of chronic arterial hypertension.. Considering the age and status of menopause, laparotomy for a left cystectomy is performed without any significant complications. On histopathological examination, the cyst was confirmed as benign serous cystadenoma of the ovary. It had a favorable postoperative evolution.
\end{abstract}

Key words: Serous cystadenoma; postmenopause; ovarian cyst(Source: MeSH NLM).

\section{INTRODUCCIÓN}

La posmenopausia constituye la etapa posterior a la fecha de última menstruación, considerada como un evento fisiológico caracterizado por la pérdida de la actividad ovárica y la interrupción permanente de la menstruación, diagnosticada después de 12 meses consecutivos de amenorrea sin una causa patológica ${ }^{1-3}$.

Pero a pesar de ello, los ovarios constituyen órganos de caracteres polimorfos, los cuales se encuentran interrelacionados entre factores genéticos, embriológicos, ontogenéticos, así como estructurales y funcionales, que interactúan entre sí para generar una enorme capacidad tumoral sea benigna o maligna ${ }^{4,5}$.

Con la edad, el ovario humano envejece en sus funciones; sin embargo, nunca pierde su capacidad para generar tumores ${ }^{6}$.

El cistoadenoma seroso de ovario es un tipo de tumor derivado del epitelio celómico superficial, formado por áreas quísticas, cuyo riesgo se incrementa con el transcurrir de la edad, ya que, pese a que la declinación

\footnotetext{
Universidad Nacional Mayor de San Marcos. Departamento Académico de Ginecología y Obstetricia. Lima- Perú.

Clínica Materno Infantil "San Pedro Norte" S. A. Lima, Perú.

Instituto Nacional Materno Perinatal. Lima-Perú

a Médico GínecoObstetra. Profesor de la Facultad de Medicina de la Universidad Nacional Mayor de San Marcos. Lima-Perú. Código ORCID https://orcid. org/0000-0002-2830-3789

Médico GínecoObstetra. Director Médico de Clínica San Pedro Norte. Lima-Perú.

c Médico Anestesiólogo. Asistente del Departamento de Anestesiología. Lima-Perú.

Médico Anatomopatóloga
}

Citar como: Citar como: Ayala-Peralta FD, Quiñones L, Sandoval L, Garrido D. Cistoadenoma seroso ovárico gigante en la posmenopausia. Reporte de un caso. Rev Peru Investig Matern Perinat 2020; 9(1): 53-8.

DOI https://doi.org/10.33421/inmp.2020190

Recibido: 15-02-20 Aprobado: 12-03-20 
de la función ovárica marca el envejecimiento gonadal, progresivo, el ovario humano nunca pierde su capacidad para generar tumores. Por lo general, cuando es detectado, su tamaño es grande, en donde la ecografía puede ayudarnos a considerar su diagnóstico ${ }^{6}$.

En estudios previos, la definición de quistes ováricos grandes o gigantes se describió como quistes que miden más de $10 \mathrm{~cm}$ de diámetro en la exploración radiológica o aquellos quistes que se extienden por encima del ombligo $^{7,8}$.

El cistoadenoma seroso ovárico de gran tamaño es raro. En la literatura, algunos casos de quistes ováricos gigantes han sido mencionado esporádicamente, principalmente en pacientes postmenopáusicas ${ }^{9,10}$

Los tumores serosos se ven comúnmente durante el período reproductivo, ocurren en adultas de todas las edades, con edades medias reportadas que difieren de 40 a 60 años; y en el $50 \%$ de ellos ocurren antes de los 40 años. La mayoría de estos quistes son de naturaleza benigna, con una probabilidad de malignidad de solo $7 \%-13 \%$ en premenopáusicas y $8 \%-45 \%$ en mujeres posmenopáusicas $^{9-11}$.

Los tumores serosos son comúnmente benignos en el $70-80 \% ; 5-10 \%$ tiene límite potencial maligno, y $20-25 \%$ son malignos. Solo el $10-20 \%$ de los casos de todos los tumores serosos son bilaterales ${ }^{10,12}$.

La mayoría de los cistoadenomas serosos son policlonales, pero ocurren cistoadenomas monoclonales; asimismo, se desarrollan como una expansión hiperplásica a partir de inclusiones epiteliales y muestran cambios en el número de copias de ADN en las células epiteliales en algunos casos $^{13}$

En cuanto a la manifestación clínica, generalmente son asintomáticas. El diagnóstico precoz es casi imposible, incluso los tumores grandes son asintomáticos y se descubren como hallazgos casuales en una exploración o ecografía. En ocasiones exhiben datos clínicos que lo hacen sospechar, como síntomas compresivos con el desplazamiento de estructuras adyacentes, crecimiento lento del abdomen, dolor pélvico abdominal subagudo ${ }^{14}$.

Para la evaluación, se requiere una buena historia clínica para explorar los síntomas y signos asociados con quistes gigantes que son inespecíficos y más comúnmente incluyen dolor pélvico, hinchazón, aumento de volumen abdominal e incomodidad ${ }^{15}$.

En relación al uso de marcador tumoral CA-125, es una herramienta útil que ayuda a distinguir entre masas ováricas benignas y malignas. Cabe mencionar que, en la mayoría de los reportes ${ }^{9,11,16,17}$ de cistoadenomas serosos, el CA- 125 estuvo dentro del rango normal o aumentó levemente.
Para el diagnóstico de cistoadenomas serosos, son útiles varias técnicas de imagen ${ }^{7,15}$, que incluyen: Ecografía transvaginal, tomografía computarizada y la resonancia magnética.

Las características más sugestivas de una neoplasia quística benigna ${ }^{15}$ incluyen: unilocularidad de quistes, septaciones mínimas, paredes delgadas y ausencia de proyección papilar.

El diagnóstico definitivo de los cistoadenomas ováricos se basa en el examen histopatológico de la muestra quirúrgica. Los hallazgos macroscópicos revelan que tienen una superficie externa lisa y contienen uno o más quistes de pared delgada llenos de líquido transparente y acuoso. Los cistoadenomas serosos generalmente son uniloculares, pero pueden ser multiloculares ${ }^{12}$.

Histológicamente, los cistoadenomas serosos están compuestos por quistes y papilas revestidos por células cuboidales a columnas no estratificadas o estratificadas que se asemejan al epitelio de las trompas de Falopio ${ }^{12}$. Por lo general, no hay atipia mínima o mínima.

El perfil inmunohistoquímico del cistoadenoma seroso es similar al del epitelio normal de la superficie ovárica y del epitelio tubárico. Además de la positividad con los marcadores epiteliales más utilizados, la p63 es positiva en la mayoría de los casos ${ }^{18}$.

Dentro de las complicaciones de los cistoadenomas ováricos $^{7}$, que son raras, incluyen: torsión ovárica y ruptura del quiste.

El manejo de los cistoadenomas ováricos ${ }^{19}$ dependen de los siguientes factores: síntomas, tamaño del quiste, edad del paciente, historial médico, estado menopáusico de la paciente. La salpingooforectomía unilateral o la quistectomía ovárica es el tratamiento adecuado de los cistoadenomas ováricos.

\section{DESCRIPCIÓN DE CASO CLÍNICO}

Se presenta el caso de una mujer de 79 años de edad, con historia ginecoobstétrica de menarquia a los 15 años de edad, fórmula obstétrica G3P3003 con partos eutócicos, última menstruación fue hace 29 años. Tiene antecedente de hipertensión arterial crónica desde hace 3 años medicado con losartan $50 \mathrm{mg}$ vía oral. Niega otros antecedentes personales y familiares; quien acude a consultorio externo de clínica San Pedro Norte por presentar dolor pélvico izquierdo irradiado a la región lumbar que se ha incrementado desde hace 4 meses de evolución. Niega otras molestias ginecológicas. Al examen físico; Presión arterial: 150/90mmHg, frecuencia cardiaca: 76/minuto, talla: $1,51 \mathrm{~m}$, peso: $52 \mathrm{~kg}$, IMC: $22,8 \mathrm{Kg} / \mathrm{m}^{2}$, circunferencia abdominal: $86 \mathrm{~cm}$. Al examen ginecológico mediante especuloscopía se evidencia trofismo vaginal 
disminuido, cérvix acortado hipotrófico adherido al fondo de saco Douglas. Al tacto vaginal; vagina reseca, acortada. Cérvix posterior $1 \mathrm{~cm}$ de tamaño longitudinal de consistencia fibromuscular. Útero anteversoflexo hipotrófico cuyas dimensiones son $6 \times 4 \times 3 \mathrm{~cm}$, no doloroso a la movilización. Anexo izquierdo ocupado por una tumoración renitente, móvil, de aproximadamente $16 \mathrm{~cm}$ que ocupa la cavidad pélvica. Resto del examen físico sin alteraciones.

Se le realizaron exámenes de laboratorio preoperatorios: Hemoglobina: 12.5 g\%, grupo sanguíneo "O" Factor Rh positivo. Glucosa $113 \mathrm{mg} \%$; urea $33 \mathrm{mg} / \mathrm{dl}$; creatinina o,68 $\mathrm{mg} / \mathrm{dl}$; tiempo de protrombina 14,9 segundos; INR 1,35; Proteína $C$ reactiva 4,83. Marcadores séricos tumorales: Antígeno carcinoembrionario (CEA): $4,3 \mathrm{ng} / \mathrm{ml}$; Ca-125: $24,74 \mathrm{U} / \mathrm{ml}$; alfafetoproteína (AFP): $5,03 \mathrm{ng} / \mathrm{ml}$. Urocultivo negativo. Electrocardiograma: ritmo sinusal. Riesgo quirúrgico II. Ecografía transvaginal: Útero mide 66x31 mm. Miometrio sin alteraciones. Endometrio lineal. Anexo izquierdo: imagen anecogénica de paredes delgadas de $79 \times 123 \times 16 \mathrm{~mm}$, volumen 550cc. Conclusión: imagen de gran volumen a descartar cistoadenoma seroso (figura 1). Tomografía espiral multicorte de abdomen y pelvis: útero de $74 \times 44 \mathrm{~mm}$. Lesión hipodensa quística de 145×101mm adyacente a la región superior de útero.

El procedimiento quirúrgico fue una laparotomía exploratoria: quistectomía izquierda. Previa asepsia y colocación de campos estériles, se procede a incisión Pfannestiel, apertura por planos, identificación de órganos pélvicos. Pinzamiento, corte y sutura de pedículo ancho del quiste (figura 2), quistectomía izquierda, revisión de hemostasia, cierre de pared por planos. El hallazgo quirúrgico fue un quiste gigante, pared delgada transparente, se visualiza contenido líquido claro y con pedículo ancho (figura 3).

El resultado anatomopatológico reporta en el examen macroscópico: una tumoración quística que pesa $794 \mathrm{~g}$ y mide $14 \times 11 \times 7,5 \mathrm{~cm}$. Superficie externa translúcida, lisa.

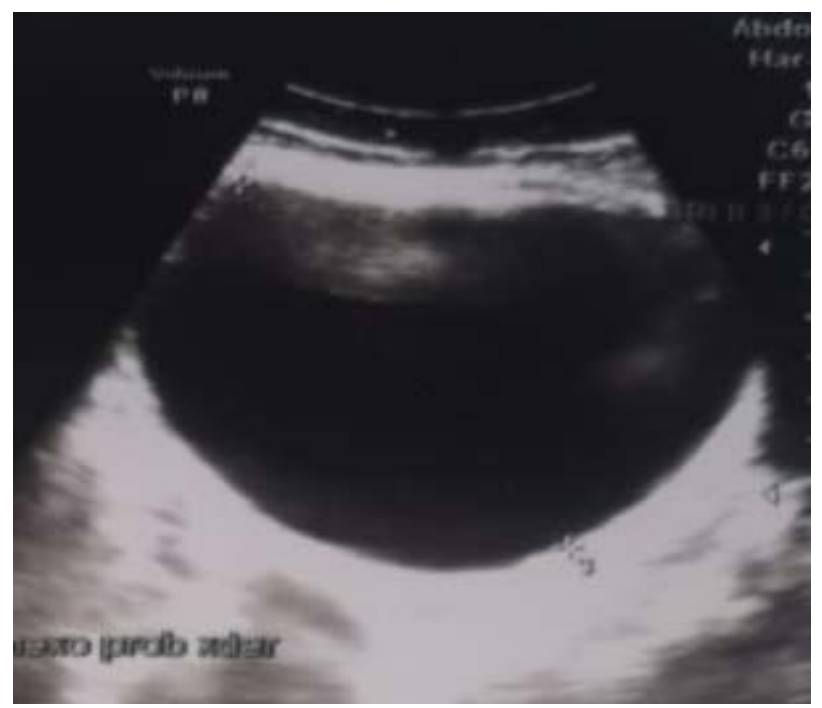

Figura 1. Ecografía transvaginal. Anexo izquierdo: imagen

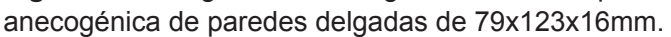

Al corte se aprecia salida de líquido seroso. Presencia de múltiples cavidades, la mayor de $14 \mathrm{~cm}$ de diámetro. Superficie interna lisa. Pared de hasta $0,2 \mathrm{~cm}$. El Diagnóstico histológico fue cistoadenoma seroso, no se observa neoplasia maligna. La evolución postoperatoria fue favorable.

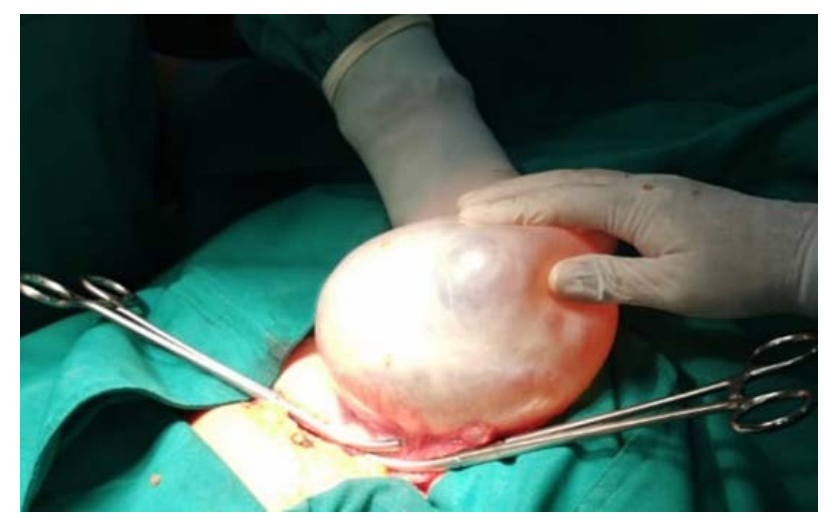

Figura 2. Pinzamiento, corte y sutura de pedículo ancho del quiste ovárica izquierda.

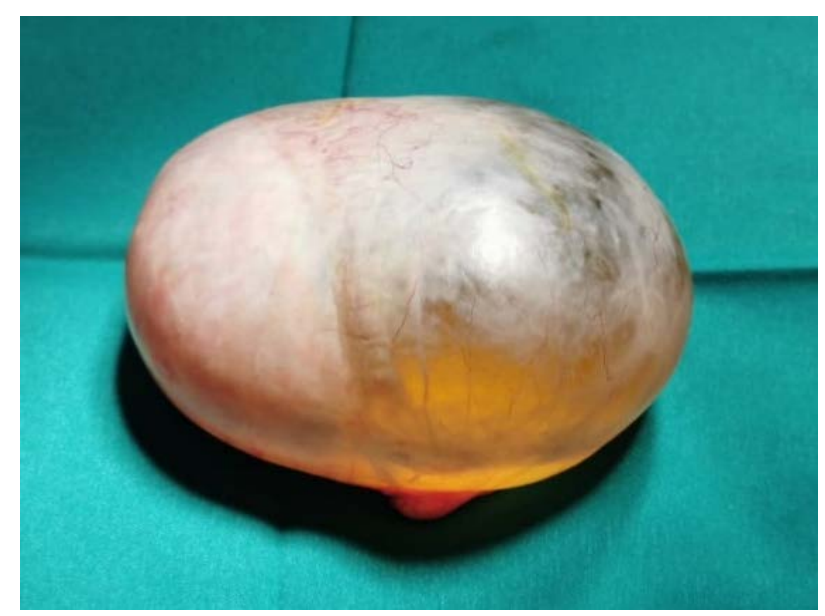

Figura 3. Imagen de quiste ovárica izquierda.

\section{DISCUSIÓN}

En el presente caso, se trata de una mujer postmenopáusica que presenta cistoadenoma seroso gigante, catalogada como tumor ovárico benigno. En la actualidad, con el uso de la ultrasonografía, el diagnóstico de tumor de ovario gigante es infrecuente, debido a la detección temprana de la patología anexial con el advenimiento de las modalidades de imágenes de rutina ${ }^{8,9}$.

Según $\mathrm{ACOG}^{20}$, la mayoría de las masas anexiales del ovario, trompas de Falopio o los tejidos circundantes, se detectan incidentalmente en el examen físico o en el momento de la imagen pélvica. Con menos frecuencia, una masa puede presentarse con síntomas de dolor agudo o intermitente. Aunque la mayoría de las masas anexiales son benignas, el objetivo principal de la evaluación 
diagnóstica es excluir la malignidad. En el presente caso, la presentación clínica fue por dolor pélvico de 4 meses de evolución irradiada a la región lumbar con aumento de volumen pélvicoabdominal y cuyo hallazgo ecográfico revela imagen quística de contenido líquido que fue confirmada por estudio histopatológico de cistoadenoma seroso de $14 \mathrm{~cm}$, que es considerada gigante por el tamaño que ocupaba la cavidad pélvicoabdominal; y el dolor se explicaría por el desplazamiento y compresión de los órganos adyacentes; tal como señalan Aggarwal A et al ${ }^{14}$, el diagnóstico precoz es casi imposible, incluso los tumores grandes son asintomáticos y se descubren como hallazgos casuales en una exploración o ecografía. En ocasiones exhiben datos clínicos que lo hacen sospechar, como síntomas compresivos, crecimiento lento del abdomen, dolor abdominal subagudo.

El cistoadenoma seroso de ovariogigante es un hallazgoraro. En la literatura, algunos casos de quistes ováricos gigantes se han mencionado esporádicamente y especialmente en mujeres posmenopáusicas de edad avanzada ${ }^{9,10}$. En el presente caso, se reporta un cistoadenoma seroso gigante de $14 \mathrm{~cm}$; tal como señalan, en estudios de Sujatha $V$ et al ${ }^{9}$, el tamaño del cistoadenoma seroso gigante fue encontrado en mujeres posmenopáusicas que miden un máximo de 60 $\times 47 \times 30 \mathrm{~cm}$.

Para el diagnóstico de tumores de ovario, se utilizan diversas técnicas de imagen, como son la ecografía pélvica, tomografía computarizada y resonancia magnética que son la elección de las modalidades de imagen que se utilizan para el diagnóstico de masas más grandes en los anexos y afectación metastásica. Además de estos, las mediciones séricas del marcador tumoral CA-125 pueden ser útiles ${ }^{8}$.

En el presente caso clínico se diagnosticó quiste seroso mediante ecografía transvaginal con hallazgos que sugirieron un quiste benigno, es decir, un quiste unilateral sin áreas sólidas o superficie irregular y sin ascitis; asimismo también se corroboró mediante la tomografía computarizada.

El valor inicial de la ultrasonografía transvaginal debe ser de primera línea para dilucidar si la tumoración ovárica quística es benigna o maligna. Para ello, los reportes de diversos autores como Sokalska $A$ et $a^{21}$, Castillo G, et $\mathrm{al}^{22}$, Ekerhovd E et $\mathrm{al}^{23}$, Modesitt SC et $\mathrm{al}^{24} \mathrm{y}$ Guraslan $\mathrm{H}$ et $\mathrm{al}^{25}$ señalan que, las características del ultrasonido de las masas benignas incluyen una apariencia simple: paredes delgadas y lisas; y la ausencia de componentes sólidos, tabiques o flujo sanguíneo interno en la ecografía doppler color. Por ello, es muy probable que estos quistes simples sean benignos en cualquier grupo de edad. Aunque como señalan Ekerhovd $\mathrm{E}$ et $\mathrm{al}^{23}$, aun no se han establecido un límite de tamaño definitivo para delinear la necesidad de intervención quirúrgica.

Pero, según reportes de Brun $\mathrm{JL}$ et $\mathrm{al}^{26}$, los quistes de 10 $\mathrm{cm}$ o más a menudo se consideran una indicación para la cirugía; sin embargo, otros autores como Pavlik EJ et al ${ }^{27}$ difieren al respecto los quistes simples (incluso los mayores de $10 \mathrm{~cm}$ ) a menudo retroceden espontáneamente cuando se examinan con ultrasonografía en serie. En nuestro caso clínico la imagen ecográfica revelaba una imagen anecogénica de paredes delgadas, volumen $550 \mathrm{cc}$, que inclinaban a un quiste seroso con características de benignidad.

En relación al uso de marcadores séricos tumorales, la mayoría de los estudios señalan que, el marcador tumoral CA-125 en pacientes con cistoadenoma seroso, estuvieron dentro del rango normal o aumentó levemente, como por ejemplo, reportan los autores Sujatha $\mathrm{V}$ et al ${ }^{9}$ el Ca-125 en 46,61 U/ml; Alobaid A et al ${ }^{11}$ como valor normal; Agrawal SP et $\mathrm{al}^{8} \mathrm{Ca}-125$ en $31,1 \mathrm{U} / \mathrm{ml}$ y $\mathrm{Kim}$ $\mathrm{HY}$ et al ${ }^{17}$ reportaron cifras de Ca-125 en $109,51 \mathrm{U} / \mathrm{ml}$. En esto contexto, el nivel de CA-125 en el presente caso clínico también estuvo dentro del valor normal, con cifras de $24,74 \mathrm{U} / \mathrm{ml}$.

En relación a las características macroscópicas y microscópicas ${ }^{12}$, el cistoadenoma seroso usualmente mide entre 3 y $10 \mathrm{~cm}$, es de forma redondeada u oval, superficie externa lisa, usualmente contienen líquido transparente o amarillento. Generalmente es unilocular, pero pueden ser multiloculares, tapizadas por una sola capa de células, columnas, ciliadas, que se asemejan al epitelio tubárico; o epitelio cuboidal no ciliado, que se asemejan a la superficie ovárica. Si presenta papilas, son simples. No se observa atipia, ni complejidad arquitectural, ni invasión.

Los quistes ováricos extremadamente grandes se manejan tradicionalmente por laparotomía. Pero los avances recientes en la cirugía endoscópica han ofrecido opciones alternativas mediante tratamiento laparoscópico ${ }^{28}$

Sin embargo, la laparotomía y la escisión total de los quistes son la opción de tratamiento en caso de quistes ováricos grandes, hasta o antes de la cirugía laparoscópica, se realiza la descompresión guiada por ultrasonido o la aspiración del quiste ${ }^{29}$. En el presente caso, se hizo laparotomía exploratoria y quistectomía.

En una revisión, Bellati $\mathrm{F}$ et $\mathrm{al}^{30}$ mencionaron la minilaparotomía guiada por laparoscopia (LGML) en caso de grandes masas anexiales benignas, sin otro factor de riesgo de malignidad, aparte del tamaño; dichos estudios concluyeron que, en términos de seguridad y viabilidad, LGML es una mejor opción en comparación con la laparoscopia.

La cirugía es esencial para los tumores de ovario grandes, incluso si es benigna. Hasta ahora, no ha habido un ensayo controlado aleatorio para el tratamiento laparoscópico de quistes ováricos $>20 \mathrm{~cm}$, por lo que la laparotomía siguió siendo el método ideal para la escisión de los quistes ováricos gigantes ${ }^{11}$. 
Asimismo señalar, que en el presente caso, el manejo fue quirúrgico mediante una laparotomía exploratoria para quistectomía por la condición de una tumoración gigante en una mujer posmenopáusica; aunque otros autores como Karpelowsky JS et $\mathrm{al}^{31}$, Savasi I et $\mathrm{al}^{32}$ y Oue $\mathrm{T}$ et $\mathrm{a}^{33}$, señalan que el tipo de intervención quirúrgica más apropiada para una supuesta masa anexial benigna fueron los procedimientos mínimamente invasivos independientemente del enfoque empleado, la preservación de la fertilidad debe ser una prioridad cuando se manejan masas en adolescentes y mujeres premenopáusicas que no han completado la maternidad; incluso en mujeres que presentan quistes ováricos grandes de $10 \mathrm{~cm}$ o más, es posible salvar porciones normales del ovario y extirpar el quiste por vía laparoscópica.

Tal como reportan Gonzalez DO et $\mathrm{al}^{34}$, el manejo de los cistoadenomas ováricos depende de los siguientes factores: síntomas, tamaño del quiste, edad del paciente, historial médico, estado menopáusico de la paciente. La salpingooforectomía unilateral o la quistectomía ovárica es el tratamiento adecuado de los cistoadenomas ováricos.

Por otro lado, es necesario recordar lo señalado por Fatema $\mathrm{N}$ et al que las complicaciones raras de los cistoadenomas ováricos incluyen, torsión ovárica y ruptura del quiste.

Finalmente señalar, que los cistoadenomas ováricos son neoplasias epiteliales benignas comunes que tienen un pronóstico excelente. En el presente caso fue un cistoadenoma seroso en una mujer posmenopáusica que tuvo evolución favorable.

Financiamiento: Autofinanciado.

Conflicto de interés: Los autores declaran no tener algún conflicto de intereses.

\section{REFERENCIAS BIBLIOGRÁFICAS}

1. Goodman NF, Cobin RH, Ginzburg SB, Katz IA, Woode DE. American Association of Clinical Endocrinologists Medical Guidelines for Clinical Practice for the diagnosis and treatment of menopause. Endocr Pract. 2011;17 Suppl 6:125

2. American College of Obstetricians and Gynacologists (ACOG). Practice Bulletin No. 141: management of menopausal symptoms. Obstet Gynecol. 2014;123(1):20216, reaffirmed 2016, correction can be found in Obstet Gynecol. 2016;127(1):166

3. Nelson HD. Menopause. Lancet. 2008;371(9614):760-70

4. Miliián I, Chávez LA, Jiménez S. Dos tipos histológicos diferentes en un tumor de ovario. A propósito de un caso. Clin Invest Gin Obst. 2015;42(2):92-94. http://dx.doi. org/10.1016/j.gine.2013.10.006.

5. Bom-Khair, Frontera DA, Eacobar ME. Estruma ovárico benigno: diagnóstico y tratamiento en adolescencia. Arch Argent Pediatr. 2005;103.
6. Fernández V, Acuña F, Recuay P, Arce K, García N, Martina M. Cistoadenoma seroso gigante. Ginecol. obstet. 2003; 49 (1) : 63-66

7. Fatema N, Mubarak Al Badi M. A Postmenopausal Woman with Giant Ovarian Serous Cyst Adenoma: A Case Report with Brief Literature Review. Case Rep Obstet Gynecol. 2018; 2018:1-4. https://doi.org/10.1155/2018/5478328.

8. Agrawal SP, Rath SK, Aher GS, Gavali UG. Large ovarian tumor: A case report. International Journal of Scientifc Study. 2015. http://www.ijss-sn.com/uploads/2/0/1/5/20153321/ ijss jun cr08.pdf.

9. Sujatha VV, Babu SC. Giant ovarian serous cystadenoma in a postmenopausal woman: A case report. Cases Journal. 2009;(7).

10. Dey M, Pathak N. Giant serous papillary cystadenoma. Medical Journal Armed Forces India. 2011;67(3):272-273.

11. Alobaid A, Memon A, Alobaid S, Aldakhil L. Laparoscopic Management of Huge Ovarian Cysts. Obstetrics and Gynecology International 2013:1-4.

12. Seidman JD, Mehrotra A. Benign ovarian serous tumors: a re-evaluation and proposed reclassification of serous "cystadenomas" and "cystadenofibromas". Gynecol Oncol. 2005;96(2):395-401.

13. Hunter SM, Anglesio MS, Sharma R, Gilks CB, Melnyk N, Chiew YE, deFazio A, Australian Ovarian Cancer Study Group. Longacre TA, Huntsman DG, Gorringe KL, Campbell IG. Copy number aberrations in benign serous ovarian tumors: a case for reclassification? Clin. Cancer Res. 2011 Dec 01;17(23):7273-82.

14. Aggarwal A, Lucco KL, Lacy J, Kives S, Gerstle JT, Allen L. Ovarian epithelial tumors of law malignant potential. A case series of 5 adolescent patients. J Pediatr Surg. 2009;44:2023-7.

15. Jeong YY, Outwater EK, Kang HK. Imaging evaluation of ovarian masses. Radiographics. 2000;20(5):1445-70.

16. Madhu Y, Harish K, and Gotam P. Complete resection of a giant ovarian tumour. Gynecologic Oncology Reports. 2013;6:4-6.

17. Kim HY, Cho MK, Bae EH, Kim SW, Ma SK. Hydronephrosis caused by a giant ovarian cyst. International Brazilian Journal of Urology. 2016;42(4): 848-849.

18. Poli Neto OB, Candido Dos Reis FJ, Zambelli Ramalho LN, Nogueira AA, de Andrade JM. p63 expression in epithelial ovarian tumors. Int. J. Gynecol. Cancer. 2006;16(1):152-5.

19. Gonzalez DO, Minneci PC, Deans KJ. Management of benign ovarian lesions in girls: a trend toward fewer oophorectomies. Curr. Opin. Obstet. Gynecol. 2017;29(5):289294.

20. ACOG. Evaluation and Management of Adnexal Masses. Practice bulletin. Number 174, November 2016.

21. Sokalska A, Timmerman D, Testa AC, Van Holsbeke C, Lissoni $\mathrm{AA}$, Leone FP, et al. Diagnostic accuracy of transvaginal ultrasound examination for assigning a specific diagnosis to adnexal masses. Ultrasound Obstet Gynecol. 2009;34:462-70.

22. Castillo G, Alcazar JL, Jurado M. Natural history of sonographically detected simple unilocular adnexal cysts in asymptomatic postmenopausal women. Gynecol Oncol. 2004;92:965-9.

23. Ekerhovd E, Wienerroith H, Staudach A, Granberg S. Preoperative assessment of unilocular adnexal cysts by transvaginal ultrasonography: a comparison between 
ultrasonographic morphologic imaging and histopathologic diagnosis. Am J Obstet Gynecol. 2001;184:48-54.

24. Modesitt SC, Pavlik EJ, Ueland FR, DePriest PD, Kryscio RJ, van Nagell JR Jr. Risk of malignancy in unilocular ovarian cystic tumors less than 10 centimeters in diameter. Obstet Gynecol. 2003;102:594-9.

25. Guraslan H, Dogan K. Management of unilocular or multilocular cysts more than 5 centimeters in postmenopausal women. Eur J Obstet Gynecol Reprod Biol. 2016;203:40-3.

26. Brun JL, Fritel X, Aubard Y, Borghese B, Bourdel N, ChabbertBuffet $\mathrm{N}$, et al. Management of presumed benign ovarian tumors: updated French guidelines.College National des Gynecologues Obstetriciens Francais. Eur J Obstet Gynecol Reprod Biol 2014;183:52-8.

27. Pavlik EJ, Ueland FR, Miller RW, Ubellacker JM,DeSimone $\mathrm{CP}$, Elder $\mathrm{J}$, et al. Frequency and disposition of ovarian abnormalitiesfollowed with serial transvaginalultrasonography. Obstet Gynecol.2013;122:210-7.

28. Sagiv R, Golan A, Glezerman M. Laparoscopic management of extremely large ovarian cysts. Obstetrics \& Gynecology. 2005;105(6):1319-1322.

29. Bhasin SK, Kumar V, Kumar R. Giant Ovarian Cyst. Case Report. 2017;16(3).
30. Bellati F, Gasparri ML, Ruscito I, Caccetta J,Benedetti Panici P. Minimal invasive approaches for large ovarian cysts: A careful choice. Archives of Gynecology and Obstetrics. 2013;287(3):615-616.

31. Karpelowsky JS, Hei ER, Matthews K. Laparoscopic resection of benign ovarian tumours in children with gonadal preservation. Pediatr Surg Int. 2009;25:251-4.

32. Savasi I, Lacy JA, Gerstle JT, Stephens D, Kives S, Allen L. Management of ovarian dermoid cysts in the pediatric and adolescent population. J Pediatr Adolesc Gynecol. 2009;22:360-4.

33. Oue T, Uehara S, Sasaki T, Nose S, Saka R, YamanakaH, et al. Treatment and ovarian preservation in children with ovarian tumors. J Pediatr Surg. 2015;50:2116-8.

34. Gonzalez DO, Minneci PC, Deans KJ. Management of benign ovarian lesions in girls: a trend toward fewer oophorectomies. Curr. Opin. Obstet. Gynecol. 2017;29(5):289-294.

\section{Correspondencia:}

Félix Dasio Ayala Peralta

Dirección: Jr. Maracaibo 2153. Lima 31

Correo: fayala1401@hotmail.com

Teléfono: 999227657 